\title{
ACOUSTICAL ABSORPTION AND SCATTERING CROSS-SECTIONS OF SPHERICAL BUBBLE CLOUDS
}

\author{
by Luca d'Agostino and Christopher E. Brennen
}

\author{
California Institute of Technology
}

Pasadena, California 91125

\section{ABSTRACT}

The present work investigates the acoustical absorption and scattering cross-sections of spherical bubble clouds subject to harmonic far field pressure excitation. Bubble dynamics effects and energy dissipation due to viscosity, heat transfer, liquid compressibility and relative motion of the two phases are included. The equations of motion for the average flow and for the bubble radius are linearized and a closed form solution is obtained. Due to the presence of natural oscillatory modes and frequencies, the acoustical crosssections of the cloud are very different from those of each individual bubble in the cloud, as well as from the acoustical cross-sections of a single large bubble with the same volume of vapor and gas. In general the acoustical properties of any given volume of the dispersed phase depend strongly on the degree of dispersion because of the complex interactions of the dynamics of the bubbles with the whole flow.

\section{INTRODUCTION}

This note illustrates part of our current research on the role played by the dynamics of bubble volume changes in the fluid mechanics of bubbly or cavitating flows and represents a natural extension of our previous work on the dynamics of one-dimensional unsteady flows of spherical bubble clouds subject to far field pressure perturbations (d'Agostino and Brennen, 1987). Among the practical objectives of this study is a better understanding of the global effects of many bubbles in the dynamics and, specifically, in the acoustical behavior of bubbly and cavitating flows. Traditionally the acoustical properties and, in particular, the noise radiation of these flows have been analysed and interpreted on the basis of single bubble dynamics assuming that the effects of individual bubbles can be algebraically summed. This assumption, for example, is inherent to virtually all commonly accepted scaling laws of noise generation in bubbly and cavitating flows; the void fraction and bubble concentration in the cavitation region never appear as scaling parameters (Blake, 1986). The interactive effects that the bubble volume changes can have in many practical cases on the velocity and pressure distributions (and therefore ultimately on the magnitude of the bubble response itself) are neglected, thus eliminating the effects of any large scale internal motion in the bubbly region of the flow.

The traditional approach may be adequate when the bubble concentration is extremely low, but it clearly loses validity when the bubble concentration becomes larger and the possibility of global motion in the bubbly mixture arises. As early as 1969 Erdmann and his co-workers noticed a surprising and unexplained sharp decrease of the sound pressure level from traveling bubble cavitation on propeller hydrofoils when the cavitation number dropped below $80 \%$ of its inception value and cavitation became more extensive. The optical observations of traveling bubble cavitation on Schiebe headforms in water tunnel tests by Marboe et al. (1986) and the simultaneous sound measurements displayed the tendency of the noise spectrum to shift towards lower frequencies than expected from single bubble dynamics considerations. Marboe and his co-workers suggested the occurrence of asymmetric bubble collapse as a possible cause of this phenomenon. In view of our current results global bubble interaction effects in the cavitation region when a sufficient concentration of bubbles is present are another possible explanation of the observed reduction of the sound pressure level and of the downward shift of the noise frequency spectrum in heavily cavitating flows. Similar recent experimental results by Arakeri and Shanmuganathan (1985) and Billet (1986) have also helped identify bubble interactions in cavitating flows as a likely source of the observed discrepancies. The main purpose of this research is to provide some physical interpretation of the origin of these alterations. Despite the extensive linearizations inherent in the analysis we are confident that the results convey a qualitative understanding of the $\mathrm{dy}$ namic and acoustical properties of real bubbly flows and represent a useful guidance in the study of such flows with non-linear bubble dynamics.

\section{DYNAMICS OF SPHERICAL BUBBLE CLOUDS}

We consider the problem of a one-dimensional flow in a spherical bubble cloud of radius $A$ and void fraction $\alpha$ located at $\underline{x}=0$ in an unbounded liquid with pressure $p$, density $\rho$, viscosity $\mu$, surface tension $S$ and speed of sound $c$, as shown in Fig. 1. Let the perturbation of the far field pressure be represented by a one-dimensional plane acoustic wave $p_{\infty}(t)=p_{o}[1-\varepsilon \exp i(\omega t-h x)]$ of wave number $h=\omega / c \ll 1 / A_{o}$ with $\varepsilon \ll 1$ and let the subscript $o$ indicate the unperturbed conditions corresponding to $\varepsilon=0$. Following the approach used in our earlier work (d'Agostino and Brennen, 1987) we seek the solution of the fluid dynamic equations for the two phases with the relevant interaction terms, namely: the liquid continuity and momentum equations, the bubble number continuity and bubble dynamic (Rayleigh-Plesset) equations and the relative motion equation for a spherical bubble of negligible mass with Stokes' viscous drag. These equations are linearized to study the propagation of spherical disturbances inside the cloud of the form $[\exp i(k r+\omega t)] / r$ which result from small changes in the bubble radius $R(r, t)$ as a function of time $t$ and the radial coordinate $r$ in the cloud. This leads to the following expression of the dispersion equation for a bubbly flow (van Wijngaarden, 1980) with the inclusion of bubble dynamic damping, liquid compressibility and relative motion effects:

$$
\frac{k^{2}}{\omega^{2}}=\left[\frac{1}{c_{m}^{2}}\left(\frac{\omega_{B}^{2}\left(1-i \omega R_{o} / c\right)}{\omega_{B \omega}^{2}-\omega^{2}+i \omega 2 \lambda}\right)+\frac{\left(1-\alpha_{o}\right)^{2}}{c^{2}}\right] /\left(1+\frac{2 \alpha_{o}\left(1-\alpha_{o}\right)}{1+9 \mu / i \omega \rho R_{o}^{2}}\right)
$$

where:

$$
\begin{gathered}
2 \lambda=\frac{4 \mu}{\rho R_{o}^{2}}+\frac{\omega^{2} R_{o}}{c}+\frac{p_{B o}}{\rho \omega R_{o}^{2}} \Im(\phi) \\
\omega_{B \omega}^{2}=\Re(\phi) \frac{p_{B o}}{\rho R_{o}^{2}}-\frac{2 S}{\rho R_{o}^{3}} \\
\phi=\frac{3 \gamma \theta^{2}}{\theta\left[\theta+3(\gamma-1) A_{-}\right]-i 3(\gamma-1)\left(\theta A_{+}-2\right)} \\
A_{ \pm}=\frac{\sinh \theta \pm \sin \theta}{\cosh \theta-\cos \theta}
\end{gathered}
$$


and $\theta=R_{o} \sqrt{(2 \omega / \chi G)}$ is the ratio of the bubble radius to the bubble thermal diffusion length. In the dispersion relation (1) $p_{B o}=p_{o}+2 S / R_{o}$ is the equilibrium pressure in the bubbles, $\omega_{B}$ is the solution of the implicit equation $\omega_{B}=\omega_{B \omega}\left(\omega_{B}\right)$ and:

$$
c_{m}^{2}=\frac{\omega_{B}^{2} R_{o}^{2}}{3 \alpha_{o}\left(1-\alpha_{o}\right)}
$$

is the low frequency sound speed in homogeneous bubbly mixtures with in compressible liquid. In the absence of damping $\omega_{B}$ reduces to the natural frequency of oscillation of a single bubble at isothermal conditions in an unbounded liquid (Plesset and Prosperetti, 1977; Knapp, Daily and Hammit, 1970). Similarly, when surface tension and energy dissipation are neglected $c_{m}$ reduces to the well known expression of the low frequency sound speed for a homogeneous mixture (van Wijngaarden, 1980). If the bubbles are in stable equilibrium in their mean or unperturbed state then $\Re(\phi) p_{B_{o}}>2 S / R_{o}$ and both $\omega_{B}$ and $c_{m}$ are real. The three terms in the effective damping coefficient $\lambda$ respectively represent the contributions of the viscous, acoustical and thermal dissipation, while $\omega_{B \omega}$ is the effective natural frequency of an individual bubble when excited at frequency $\omega$ and $\Re(\phi) / 3$ can be interpreted as the equivalent polytropic exponent of the gas in the bubble, which respectively tends to 1 and $\gamma$ in the isothermal and isentropic limits for $\omega \rightarrow 0$ and $\omega \rightarrow+\infty$ (Prosperetti, 1984).

Applying the conditions that the flow must be regular at the center of the cloud and that the pressure $p$ and the radial velocity $u$ of the liquid must be continuous at the cloud boundary ( $r \simeq A_{o}$ in the linearized approximation) the solution for the flow inside the bubble cloud (d'Agostino and Brennen, 1987) is:

$$
\begin{gathered}
R(r, t)=R_{o}-R_{o} \varepsilon \frac{p_{o}\left(1-\alpha_{o}\right) / \rho R_{o}^{2}}{\omega_{B \omega}^{2}-\omega^{2}+i \omega 2 \lambda} \times \\
\left(\frac{1-i \omega R_{o} / c}{\cos k A_{o}-\alpha_{o} \sin \left(k A_{o}\right) / k A_{o}}\right) \frac{\sin k r}{k r} e^{i \omega t} \\
p(r, t) \approx p_{o}+p_{o} \varepsilon \frac{1-\alpha_{o}}{\cos k A_{o}-\alpha_{o} \sin \left(k A_{o}\right) / k A_{o}}\left(\frac{\sin k r}{k r}\right) e^{i \omega t} \\
u(r, t)=i \varepsilon \frac{p_{o} / \rho \omega r}{\cos k A_{o}-\alpha_{o} \sin \left(k A_{o}\right) / k A_{o}}\left(\cos k r-\frac{\sin k r}{k r}\right) e^{i \omega t}
\end{gathered}
$$

where $k$ is the principal square root (with non-negative real and imaginary parts) of $k^{2}$ given by the dispersion equation ( 7 ).

For comparison, the solution for a single bubble of equilibrium radius $\boldsymbol{R}_{o}$ subject to the same acoustic field is (Prosperetti, 1984):

$$
\begin{gathered}
p_{R}(t)=p_{o}+\epsilon \frac{p_{o}}{\rho R_{o}^{2}}\left(p_{B o} \phi-\frac{2 S}{R_{o}}+i \omega 4 \mu\right) \frac{1-i \omega R_{o} / c}{\omega_{B \omega}^{2}-\omega^{2}+i \omega 2 \lambda} e^{i \omega t} \\
u_{R}(t)=\dot{R}(t)=-i \omega \varepsilon\left(\frac{p_{o}}{\rho R_{o}}\right) \frac{1-i \omega R_{o} / c}{\omega_{B \omega}^{2}-\omega^{2}+i \omega 2 \lambda} e^{i \omega t}
\end{gathered}
$$

where $p_{R}(t)$ and $u_{R}(t)=\dot{R}(t)$ are respectively the pressure and the velocity of the liquid at the bubble surface.

Now, the average power absorbed by the forced oscillations of a sphere (whether a cloud or a single bubble) with unperturbed radius $b_{o}$ during a period $T=2 \pi / \omega$ of the exciting acoustic field is:

$$
\dot{W}_{a}=-\frac{1}{T} \int_{0}^{T} 4 \pi b_{o}^{2} p_{b}(t) u_{b}(t) d t
$$

where $p_{b}(t) t$ and $u_{b}(t)$ are the pressure and the velocity at the sphere boundary.

The oscillating sphere also acts as a monopole source which generates the acoustic field:

$$
\begin{gathered}
p^{\prime}(r, t)=i \frac{\rho U_{b} \omega b_{o}^{2}}{\left(1+i h b_{o}\right) r} e^{i\left[\omega t-h\left(r-b_{o}\right)\right]} \\
u^{\prime}(r, t)=i \frac{U_{b} h b_{o}^{2}}{\left(1+h b_{o}\right) r}\left(1+\frac{1}{i k r}\right) e^{i\left[\omega t-h\left(r-b_{o}\right)\right]}
\end{gathered}
$$

Hence the average power radiated by the sphere is:

$$
\dot{W}_{\sigma}=\lim _{r \rightarrow+\infty} \frac{1}{T} \int_{0}^{T} 4 \pi r^{2} p^{\prime}(r, t) u^{\prime}(r, t) d t
$$

Normalization of $\dot{W}_{a}$ and $\dot{W}_{\sigma}$ with the average power density $\varepsilon^{2} p_{o}^{2} / \rho c$ of the excitation wave gives the following expressions for the acoustical absorption and scattering cross-sections of an oscillating sphere:

$$
a=-4 \pi b_{o}^{2} \frac{\Re\left(P_{b}\right) \Re\left(U_{b}\right)+\Im\left(P_{b}\right) \Im\left(U_{b}\right)}{\varepsilon^{2} p_{o}^{2} / \rho c}
$$

$$
\sigma=\lim _{r \rightarrow+\infty} 4 \pi r^{2} \frac{\Re\left(P^{\prime}(r)\right) \Re\left(U^{\prime}(r)\right)+\Im\left(P^{\prime}(r)\right) \Im\left(U^{\prime}(r)\right)}{\varepsilon^{2} p_{o}^{2} / \rho c}
$$

where $P_{b}, U_{b}, P^{\prime}(r)$ and $U^{\prime}(r)$ respectively indicate the complex amplitudes of $p_{b}(t), u_{b}(t), p^{\prime}(r, t)$ and $p^{\prime}(r, t)$

The acoustical cross-sections of either a bubble cloud or of a single bubble can then be computed from the above formulas. Clearly, for a bubble cloud $b_{o}=A_{o}$, while $p_{b}(t)$ and $u_{b}(t)$ are given by equations (8) and (9) for $r=A_{0}$. On the other hand, for a single bubble $b_{o}=R_{o}$, with $p_{b}(t)$ and $u_{b}(t)$ expressed by equations (10) and (11).

\section{RESULTS AND DISCUSSION}

In this Section we consider the case of air bubbles $\left(\gamma=1.4, \chi_{G}=\right.$ $\left.0.0002 \mathrm{~m}^{2} / \mathrm{s}\right)$ in water $\left(\rho=1000 \mathrm{~kg} / \mathrm{m}^{3}, \mu=0.001 \mathrm{Ns} / \mathrm{m}^{2}, S=0.0728 \mathrm{~N} / \mathrm{m}\right.$ $c=1485 \mathrm{~m} / \mathrm{s}$ ). Unless otherwise specified the remaining flow parameters are: $p_{o}=10^{5} \mathrm{~Pa}, R_{o}=0.001 \mathrm{~m}, A_{o}=0.1 \mathrm{~m}$ and $\varepsilon=0.1$. In most cases the parameter $\omega_{B}^{2} A_{o}^{2} / c_{m}^{2}=3 \alpha_{o}\left(1-\alpha_{o}\right) A_{o}^{2} / R_{o}^{2}$ is assigned and the void fraction $\alpha_{o}$ is determined accordingly.

Free oscillations of the cloud only occur in the absence of damping when the exciting frequency $\omega$ experienced by each bubble is equal to the natural frequency $\omega_{B}$ of an individual bubble in an infinite liquid (bubble resonance condition) or to one of the natural frequencies of the bubble cloud. In the limit of low void fraction the natural frequencies $\omega_{n}$ of the cloud are approximated by the infinite sequence;

$$
\omega_{n}^{2} \simeq \omega_{B}^{2} /\left(1+\frac{3 \alpha_{o}\left(1-\alpha_{o}\right) A_{o}^{2}}{(n-1 / 2)^{2} \pi^{2} R_{o}^{2}}\right) ; \quad n=0,1,2, \ldots
$$

For large $n$ this sequence converges to the frequency $\omega_{B}$ corresponding to the bubble resonance conditions. For small $n$ the behavior of this sequence depends on the value of $3 \alpha_{o}\left(1-\alpha_{o}\right) A_{o}^{2} / R_{o}^{2}=\omega_{B}^{2} A_{o}^{2} / c_{m}^{2}$. When this parameter is of order unity or larger the lowest natural modes can occur at comparatively low frequency. When the reverse is the case all the natural modes of the system take place with a frequency only slightly lower than the bubble resonance frequency. The occurrence of resonances in the cloud also divides the flow solution into three different regimes, namely: sub-resonant $\left(0<\omega<\omega_{1}\right)$, trans-resonant $\left(\omega_{1}<\omega<\omega_{B}\right)$ and super-resonant $\left(\omega>\omega_{B}\right)$. As we shall see later, this has significant consequences on the behavior of the flow.

The relative amplitudes of the bubble radius oscillations at the center and at the surface of the cloud are shown in Fig. 2 as a function of the normalized square frequency for a typical case of $3 \alpha_{0}\left(1-\alpha_{0}\right) A_{0}^{2} / R_{0}^{2}=\pi^{2} / 4$. At the boundary of the cloud all resonance peaks except the first are virtually eliminated by the presence of damping and replaced by a second much smaller and broader peak around the individual bubble natural frequency. At the center of the cloud the peak corresponding to the second resonant mode (whose amplitude is larger in the inner regions of the cloud) is still recognizable, although greatly attenuated. On the other hand, the peak at the bubble resonance frequency is absent because it is not associated with any global motion in the flow and because any external disturbance at the bubble natural frequency is quickly attenuated by the resonant response of the bubbles in the outer regions of the cloud. Also note that the amplitude of the bubble radius response is larger at the center of the cloud than at the surface. The other flow variables behave in a qualitatively similar manner (d'Agostino and Brennen, 1987). Therefore the first natural mode of oscillation of the cloud at a frequency $\omega \simeq \omega_{1}$ represents the most important component of the cloud response. Its effects also dominate the contributions of individual bubbles at their own natural frequency. The above results clearly indicate that the acoustical properties of bubble clouds are not adequately described in terms of the independent responses of individual bubbles, at least as long as the parameter $3 \alpha_{o}\left(1-\alpha_{o}\right) A_{o}^{2} / R_{o}^{2}$ is of order one or larger and therefore the first natural frequency of the cloud is significantly smaller than $\omega_{B}$.

The relative amplitudes, at various frequencies, of the damped bubble radius oscillations as a function of $r / A_{o}$ are illustrated in Fig. 3 for $3 \alpha_{o}(1-$ $\left.\alpha_{o}\right) A_{o}^{2} / R_{o}^{2}=\pi^{2} / 4$. Note that the bubble response is larger at the center of the cloud for forcing frequencies below the bubble natural frequency, while the reverse is the case for super-resonant excitation. In fact, in the subresonant regime the bubbles have ample time to react and therefore behave in a compliant way, with the largest motion concentrated in the interior of the cloud. The pressure change is essentially in phase with the excitation and the bubble response is almost in phase opposition. On the other hand, in super-resonant flows the bubbles cannot respond as quickly as the excitation requires because of their inertia and therefore appear to be "stiffer". This effect clearly increases with the excitation frequency and therefore the cloud response, initially concentrated in the outer regions, becomes more uniform at higher frequencies. The pressure and the bubble radius changes are almost in phase with the excitation. Finally, in the trans-resonant regime the situation is complicated by the presence of more articulated internal motions of the cloud due to the occurrence of resonances. The phase of the flow parameters with respect to the excitation depends on the dominant oscillation mode in the cloud. Between the first and the second natural frequencies, for example, 
the bubble radius response is essentially in phase with the excitation, while the pressure is almost in phase opposition.

The effects of different void fractions are illustrated in Figs. 4 and 5 , which show the acoustical absorption and scattering cross-sections of a bubble cloud as a function of the normalized square frequency for various values of the parameter $3 \alpha_{o}\left(1-\alpha_{o}\right) A_{o}^{2} / R_{o}^{2}$. Note the presence of two peaks corresponding to the first and the second natural modes of the bubble cloud and the absence of a third peak at bubble resonance conditions. Since the natural frequencies are determined by the parameter $3 \alpha_{o}\left(1-\alpha_{o}\right) A_{o}^{2} / R_{o}^{2}$ through equation (17), the peak frequencies corresponding to the cloud's natural modes of oscillations decrease at higher void fractions. Also note that the maximum values of the acoustical absorption and scattering cross-sections increase slightly with void fraction and the second resonant peaks tend to become more pronounced due to the greater compressibility of the cloud.

Comparisons of the acoustical absorption and scattering cross-sections of a bubble cloud with those of each individual bubble in the cloud and of a single large bubble with the same total volume as the bubbles in the cloud are shown in Figs. 6 and 7 for $3 \alpha_{o}\left(1-\alpha_{o}\right) A_{o}^{2} / R_{o}^{2}=\pi^{2} / 4$. Note that the results in the various cases vary by orders of magnitude in both amplitude and spectral distribution. In particular, the large bubble has the highest acoustical absorption cross-section with the lowest peak frequency. On the other hand, the individual bubble has a lower maximum value of the acoustical absorption cross-section, but the highest peak frequency, while the bubble cloud is characterized by an intermediate value of the peak frequency and by a much smaller maximum amplitude of the acoustical absorption crosssection. The situation for the acoustical scattering cross-sections is similar, with the peaks located at the same frequencies. However, the spread in the maximum values is significantly reduced because the scattering cross-section of the bubble cloud is larger than that of the individual small bubble and only slightly lower that the scattering cross-section of the single large bubble. It appears therefore that the acoustical properties of any given volume of the dispersed phase depend strongly on the degree of dispersion in the bubbly mixture. This has important consequences in the analysis of noise in bubbly and cavitating flows.

Clearly, the validity of the previous theory is limited by the various simplifying assumptions that have been made. The discussion of these assumptions is beyond the scope of this short note. Here we simply indicate that the nost important restrictions are due to the introduction of the continuum model of the flow, to the use of the linear perturbation approach in deriving the solution and to the neglect of the local pressure perturbations in the neighborhood of each individual bubble. The implications of the above approximations are examined in our previous work on the dynamics of bubble clouds (d'Agostino and Brennen, 1987).

\section{CONCLUSIONS}

The results of this study reveal a number of important effects occurring in confined bubbly and cavitating flows. As anticipated in the introduction and confirmed by the present theory, the dynamics of the bubbles is strongly coupled through the pressure and velocity fields with the global dynamics of the flow in the bubble cloud. The bubbles are responsible for the occurrence of bubble resonance phenomena and for the drastic modification of the sonic speed in the medium, which decreases and becomes dispersive (frequency dependent). Furthermore, internal resonant modes of oscillation are possible at the system's natural frequencies due to the presence of boundaries confining the bubbly region of the flow.

The occurrence of resonances leads in turn to the identification of three different flow regimes, referred to as sub-resonant, trans-resonant and superresonant. These are defined by the relation between the exciting frequency and the first natural frequency of the cloud and the individual bubble natural frequency. The natural frequencies of the cloud are always lower than the natural frequency of the individual bubbles. In particular, they become significantly smaller than the bubble resonance frequency when the parameter $3 \alpha_{o}\left(1-\alpha_{o}\right) A_{o}^{2} / R_{o}^{2}=\omega_{B}^{2} A_{o}^{2} / c_{m}^{2}$ is of order unity or larger. In the presence of damping the first natural mode of oscillation of the cloud is the most important component of the cloud dynamic response. Its effects dominate those of higher modes and the contributions of individual bubbles at their own natural frequency. Substantial global bubble interactions occur in the flow, with the result that the acoustical properties of bubbly clouds are no longer adequately described in terms of the collective but independent responses of the individual bubbles. In particular, the acoustical absorption and scattering cross-sections of a bubble cloud are very significantly different in both amplitude and frequency distribution from the acoustical absorption and scattering cross-sections of individual bubbles in the cloud. They are also very different from the cross-sections of a single large bubble with the same total volume of vapor and gas. It appears therefore that the acoustical properties of any given volume of the dispersed phase depend strongly on the degree of dispersion of the vapor/gas phase in the bubbly mixture. An increase of the void fraction also causes a substantial reduction in the amplitude of the bubble response. This, in turn, could reduce the acoustic noise in bubbly mixtures or the damage potential in cavitating flows. The above phenomena may help to explain soine of the unexpected changes experimentally observed in the noise spectrum of bubbly cavitating flows.

The present theory contains many simplifying assumptions involving the flow geometry and the linearization of both the velocity field and the bubble dyn amics. It cannot, therefore, be expected to provide a quantitative description of the unsteady behavior of bubble clouds subject to far field pressure excitation, except in the acoustical limit. Large bubble radius perturbations occur in most flows of practical interest; hence the most crucial limitation in the present paper is the linearization of the bubble dynamics, while the assumption of small velocity perturbations is likely to be more widely justified. If all the above linearizations were omitted, only numerical solutions could be realistically attempted. However, if only the hypothesis of linear bubble dynamics is relaxed, the development of quasi-linear theories might be possible and would have a much broader applicability.

\section{ACKNOWLEDGEMENTS}

The authors would like to thank Cecilia Lin for her help in drawing the pictures. This work was supported by the Naval Sea System Command General Hydromechanics Research Program Administered by the David Taylor Naval Ship Research and Development Center under Contract No. N0016785-K-0165, by the Office of Naval Research under contract No. N0014-83-K0506 and by a Fellowship for Technological Research administered by the North Atlantic Treaty Organization - Consiglio Nazionale delle Ricerche, Italy, Competition No. $215.15 / 11$ of 11.5 .1982 . Their support is gratefully acknowledged.

\section{REFERENCES}

Arakeri V. H. and Shanmuganathan V., 1985, "On the Evidence for the Effect of Bubble Interference on Cavitation Noise", J. Fluid Mech., Vol. 159, pp. 131-150.

Billet M., 1986. Personal communication.

Blake W.K., 1986, "Mechanics of Flow-Induced Sound and Vibration", Vol. 1 and 2, Academic Press, Inc.

d'Agostino L., Brennen C.E., 1987, "Linearized Dynamics of Spherical Bubble Clouds" , in preparation for submission to the Journal of Fluid Mechanics.

Erdmann H., Hermann D., Norsback M., Quinkert R. and Sudholf H., 1969, "Investigation of the production of noise by the propeller particularly with regard to the combined acoustic problem-work segments II and III", Battelle Institute E.V. Frankfurt Am Main, April 30, 1969

Knapp R. T., Daily J. W. and Hammit F. G., 1970, "Cavitation", McGraw Hill.

Marboe R.C., Billet M.L. and Thompson D.E., 1986, "Some Aspects of Traveling Bubble Cavitation and Noise”, Int. Symp. on Cavitation and Multiphase Flow Noise, Anaheim, California.

Plesset M. S. and Prosperetti A., 1977, "Bubble Dynamics and Cavitation", Ann. Rev. Fluid. Mech., Vol. 9, pp. 145-185.

Prosperetti A., 1984, "Bubble Phenomena in Sound Fields: Part One", U1trasonics, March 1984, pp. 69-78.

van Wijngaarden L., 1980, "Sound and Shock Waves in B.bubbly Liquids", Cavitation and Inhomogeneities in Underwater Acoustics, Springler-Verlag, pp. $127-140$

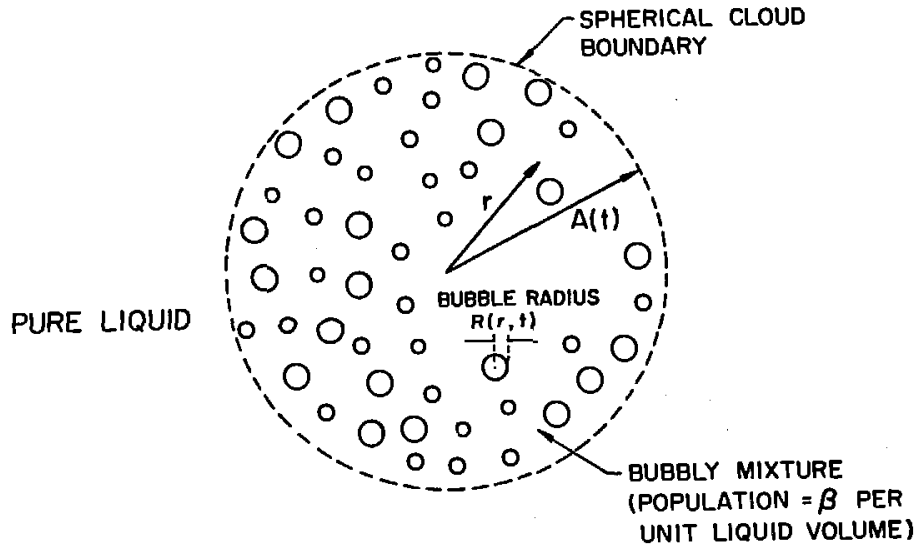

Figure 1. Schematic of a spherical cloud of bubbles. 


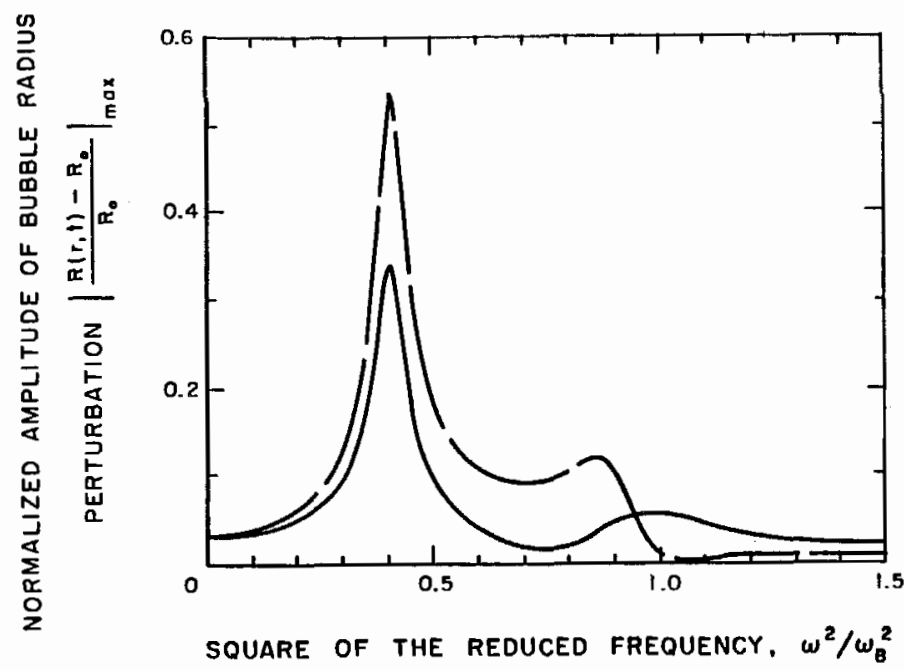

Figure 2. Normalized amplitude of the bubble radius damped oscillations at the surface $\left(r=A_{o}\right.$, solid line) and at the center of the cloud $(r=$ 0 , broken line) as a function of the square reduced frequency $\omega^{2} / \omega_{B}^{2}$ for $3 \alpha_{o}\left(1-\alpha_{o}\right) A_{o}^{2} / R_{o}^{2}=\pi^{2} / 4$.

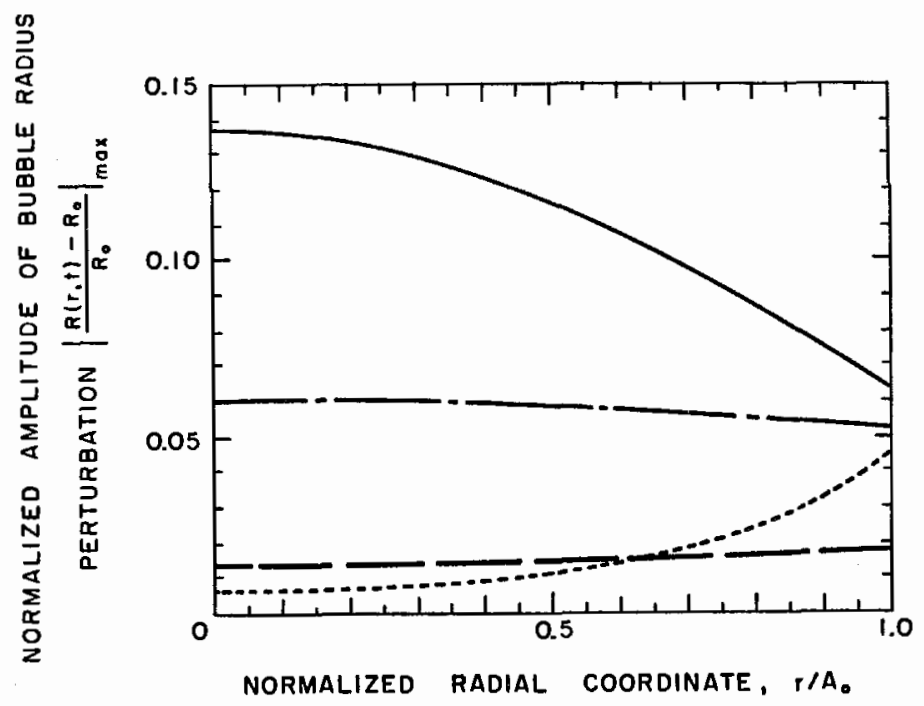

Figure 3. Normalized amplitude of the bubble radius damped oscillations in a bubble cloud as a function of the normalized radial coordinate $r / A_{o}$ for $3 \alpha_{o}\left(1-\alpha_{o}\right) A_{o}^{2} / R_{o}^{2}=\pi^{2} / 4$ and various values, of the excitation frequency: $\omega^{2}=\omega_{1}^{2} / 2$ (solid line), $\left(\omega_{1}^{2}+\omega_{2}^{2}\right) / 2$ (dash-dotted line), $1.1 \omega_{B}^{2}$ (dotted line) and $2 \omega_{B}^{2}$ (broken line). 

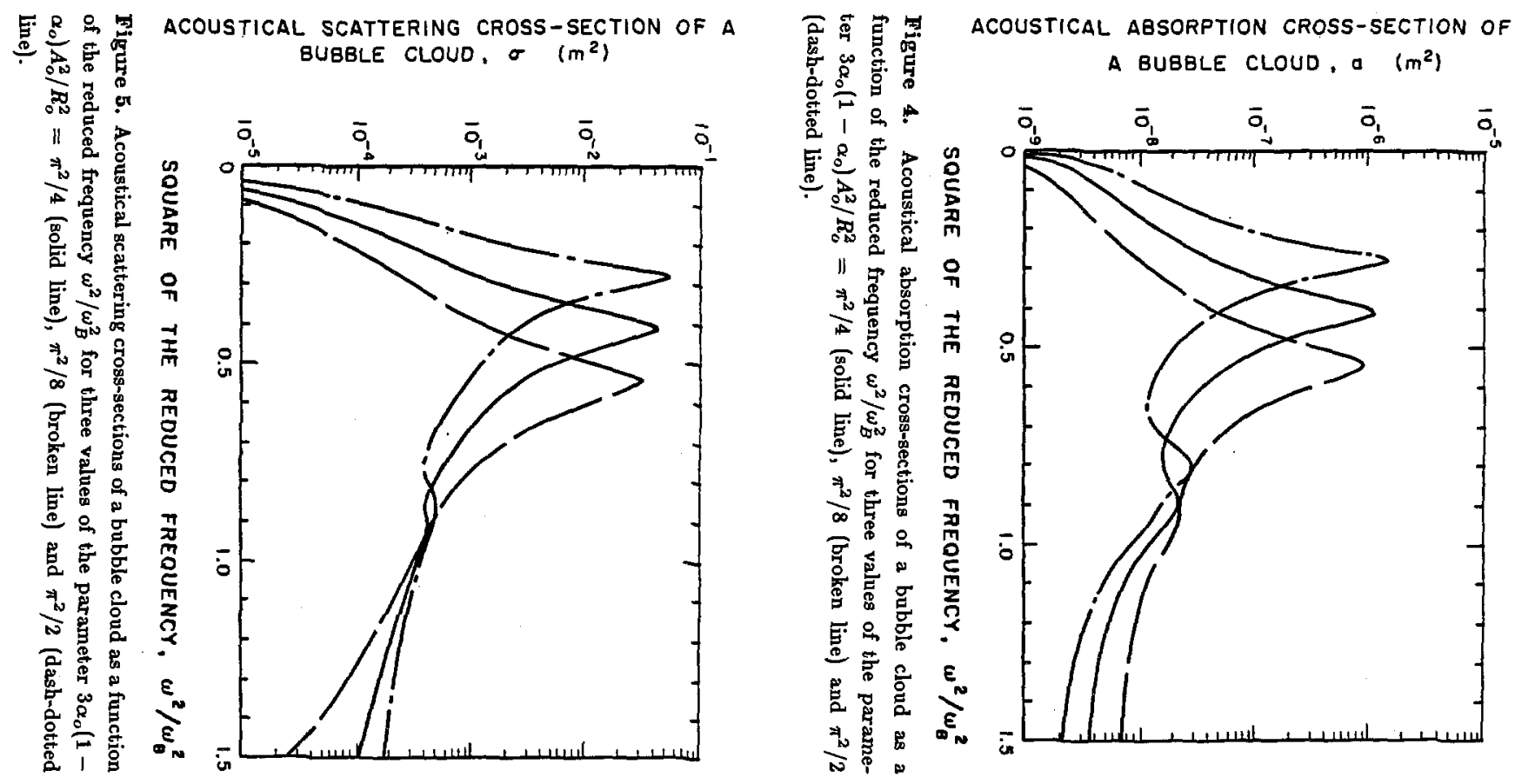

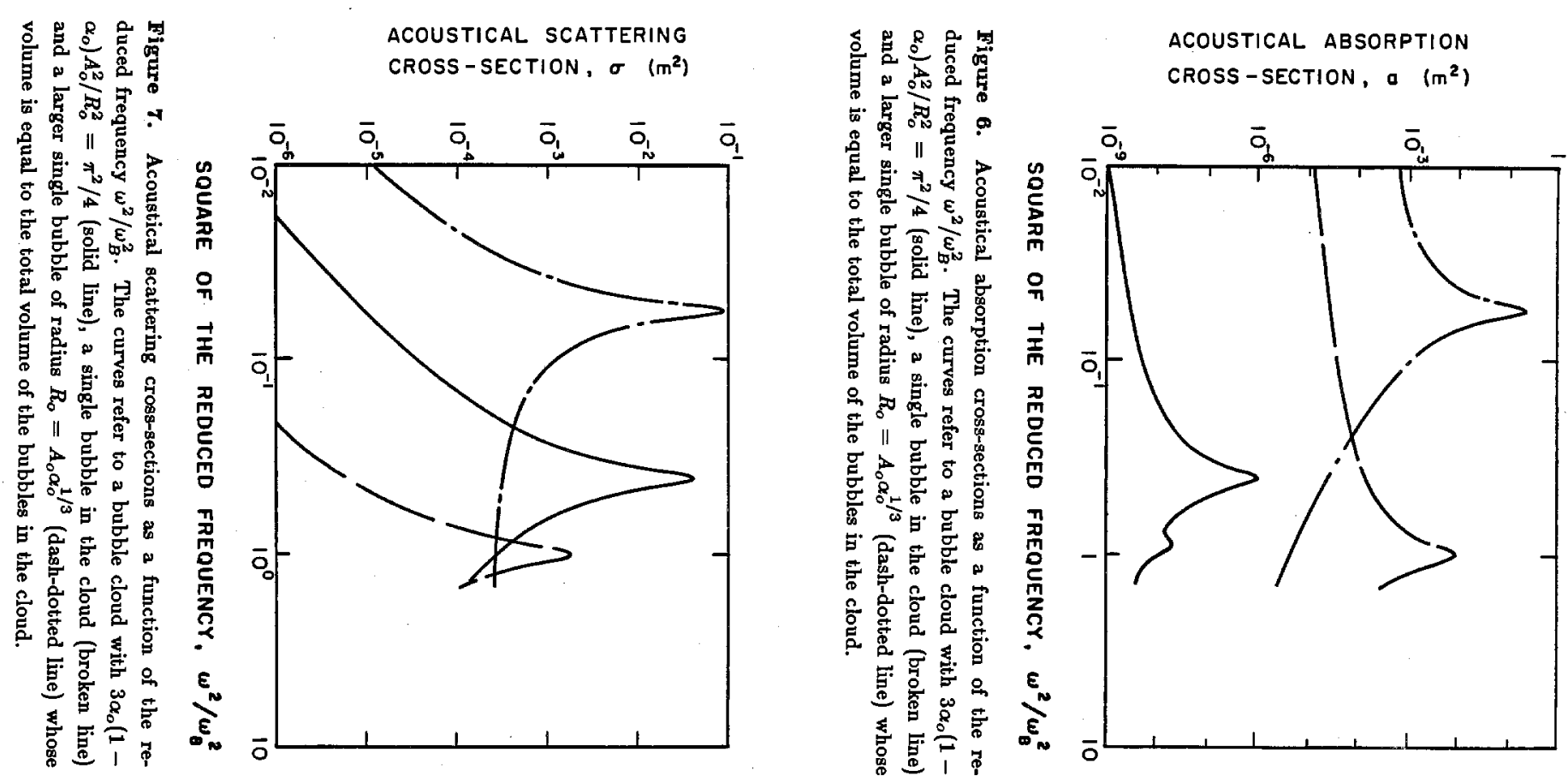